\title{
To Arrive At Benchmarking For Through Evaluation of Job Performance and Behavioural Assessment
}

\author{
Dr Shubhakar Gajula $^{1 *}$, Prof. Beena Chintalapuri ${ }^{2}$
}

\section{ABSTRACT}

This is an exploratory study aimed to examine the relationship between the behavioural traits and supervisor rating of performance of Sales Representatives in a Beverage Company in Asia inorder to arrive at a benchmark for employee development. A sample of 73 employees in the sales representative position responded to Harrison Assessments Work Preferences Inventory (Harrison, Dan; 1997). Of the 175 Harrison Assessments' traits, 39 traits showed a relationship with job performance ratings given by the supervisors. Ten of the 39 traits showed a significant linear correlation with job performance scores. The overall Suitability Score generated for Sales Representatives had a 0.633 correlation with actual job performance of Sales Representatives. This shows that the traits reflected in this study may be used as one of the pointers in selection criteria and also a key feature in the process of selection.

Keywords: Benchmarking, evaluation, Job Performance, Behavioural Assessment

A sale is a critical component in any organization structure and has a direct impact on the revenues of the company. In sales related occupation, some of the personality traits (eg Diplomatic, Outgoing, Helpful), more than others, are likely to be associated with successful performance. However, a trait list could be arrived at by assessing existing employees with high performance as their track record in achieving the job objectives in sales. Using their profile (traits) as a benchmark could help in screening potential employees during recruitment process. It may be pointed out here that candidates who attend recruitment may not show a very high level of traits required for the job but the presence of optimum level of these traits could contribute a great deal to accelerate individual and organizational progress.

It is essential to every organization to measure performance against a recognised standard so as to derive benefits and to improve the performance. Such an organizational effort is towards the process of reengineering or quality improvement initiative for enhancing the performance. To

\footnotetext{
${ }^{1}$ Assessment Researcher, Harrison Assessment International, Hyderabad

${ }^{2}$ Co-ordinator, Sahayam Counselling Centre, Osmania University, Hyderabad

*Responding Author

(C) 2016 I S Gajula, B Chintalapuri; licensee IJIP. This is an Open Access Research distributed under the terms of the Creative Commons Attribution License (http://creativecommons.org/licenses/by/2.0), which permits unrestricted use, distribution, and reproduction in any Medium, provided the original work is properly cited.
} 
enable this process of reengineering; it is important to identify the high performing employees and study the factors (behavioural \& Cognitive) that are contributing to this high performer (Sales Report, 2015). High performers are worthy to be showcased as "Models" to other performers and 'Derive a recruitment framework' so as to select individuals who show optimum or high scores on the traits which are seen in the high performers is the authors credence.

Barrick and Mount's (1991) meta analysis work suggests that the concept of personality testing is a valid predictor of job performance. Further, research has shown evidence that there exists a potential relationship between the famous Five-Factor Model (FFM) and overall job performance.

Research also suggested that personality traits as predictor variables can be generalized across all occupations and work tasks (Barrick \& Mount, 1991). However, the limitation has been that only 5 factors could be assessed using FFM and other relevant traits are ignored or failed to be assessed.

The concept of benchmarking is brought in here as it is the core component of the present study. The process of arriving at benchmarking involved assessment of personality traits but also was linked to organization's sales committee's actual evaluation of performance and their agreement or acceptance of the base performance and benchmarking level of performance. In this process of benchmarking, two evaluations i.e personality assessment and actual evaluation of job performance were blind to each other and agreed on actual. The model of benchmarking followed was Deming's model (Deming, 1950). This was the methodology followed for the present study.

The present study focused on identifying the personality traits in sales representatives using Benchmarking process. Benchmarking is part of Total Quality Management (TQM), a subject characterized by the culture of continuous improvement. According to American Productivity and Quality Centre (APQC), benchmarking is a process of identifying, understanding and adapting outstanding practices and processes from organization anywhere in the world by a firm to improve its performance” (APQC, 1993). The exceptional performance is identified by measures of performance indicators, which are called benchmarks and the activities that facilitate the exceptional performance called "Enablers". Enablers explain the reason for the superior performance; therefore benchmarking studies are conducted with the support of the two components when they are practically connected. Most of the approaches take their root in an iterative benchmarking process proposed by W.E.Deming, famously referred as "Deming Cycle" - it includes four phases "Plan-Do-Action-Check" (Deming, 1950).

To identify the relationship between the personality traits and job performance, the researcher has used Harrison benchmarking technology for the present study. Harrison benchmarking technology is a software program that utilizes a proprietary algorithm to identify the suitability traits measured by the Harrison Suitability Assessment that demonstrate a relationship with the 
overall performance score. It also identifies the relative importance of the trait relationships. The 175 traits considered in the analysis included a full range of suitability factors related to personality, attitudes, motivations, interpersonal skills, task preferences, interests, and work environment preferences.

Harrison Assessments Benchmarking Technology generates a Job Success Formula (JSF) which formulates the traits related to success. The traits are formulated by awarding either positive or negative points to different levels of the traits based on the relative importance of each trait to performance and how each level of each trait impacts performance.

The traits are categorized as Essential traits, Desirable traits and Traits to avoid or Negative Traits. Essential traits demonstrate a linear relationship with performance. Desirable traits and traits to avoid, demonstrate a nonlinear relationship with performance.

The Job Success Formula (JSF) is used to calculate an overall suitability score based on the trait scores of individuals and how closely their responses align with the Job Success Formula (JSF). The overall suitability score is a percentage based on the sum of the points earned by the individual divided by the number of points possible in the Job Success Formula (JSF). The overall suitability score is then interpreted in the following manner:

90-100 Expected to be an excellent performer

80-89 Expected to be a good performer

75-79 Expected to be an average performer

70-74 Expected to be a below average performer

69 or below Expected to be a poor performer

\section{METHOD}

\section{Objective}

- $\quad$ To identify the personality traits measured by the Harrison Suitability Assessment

- $\quad$ To measure the relationship between HA traits and overall Job performance score

- $\quad$ To arrive at norms for the group and a benchmarked Job Success Formula template that could be used by the organization under study

\section{Sample}

The sample consisted of 73 employees who were holding Sales Representative position. Entire sample is a homogeneous with similar responsibilities. Individuals of the sample had an average tenure of 5 and half years as sales representatives. Out of the 73 employees 32 persons have a work experience of 6-10 years in the same position and same company.

Other demographic details of the sample are not disclosed inorder to maintain confidentiality. 


\section{Description of the tool}

Two tools were used;

- $\quad$ Harrison Assessments Suitability Questionnaire

- Job Performance evaluation data sheet/Scale

\section{Harrison Assessments Suitability Questionnaire}

The author of the test is Dr. Dan Harrison, founder and CEO of Harrison Assessments International Limited. The Harrison Assessments Suitability Questionnaire is an online test and measures 175 suitability traits, 30-40 of which apply to any job function. The test has statements arranged in 18 frames and could be completed in approximately 30 minutes. The first 16 frames have 8 statements in each frame and the respondent has to arrange the statements as per their priority or experience. The $17^{\text {th }}$ frame is related to capture the interest and the $18^{\text {th }}$ frame has 8 statements to be value-arrange.

Harrison Assessments has Job Success Formulas that relate to more than 650 job titles, each of which offer up to four levels of experience and three levels of management. Since, eligibility and suitability are different for each job level and each management level, the assessment factors are adjusted in each case making a total of over 6500 Job Success Formulas. The Job Success Formulas are based on 20 years of performance research and thus accurately reflect the behavioural requirements and scoring. The suitability formulas can be easily customized according to the specific behavioural requirements of the job. The norms are developed based data of more than 10,00,000 respondents across various countries. It is to be noted that the norms are used diligently keeping in view and culture and country for interpretation.

Job Success Formula templates gives three set of trait list:

a. Essential Traits: These are traits in which higher scores generally relate better performance.

b. Desirable Traits: These are traits in which low scores hinder success but there is not necessarily a significant overall correlation with performance. In other words, poor performers have a higher incidence of low scores on this type of trait.

c. Traits to Avoid: These are traits that hinder success but don't necessarily have a negative correlation with success

\section{Job Performance scale}

The tool was a rating scale designed by the sales department of the organization. Each employee was rated by the Sales department according to their actual on job performance. Each member of the sample was rated by their supervisor. The criteria used for evaluation is given in table1 


\begin{tabular}{|l|l|l|}
\hline S.No & Performance Criteria & Weight age \\
\hline 1 & Monthly Volume Hit rate & $60 \%$ \\
\hline 2 & Order Placement Activeness & $0 \%$ \\
\hline 3 & Refrigerator Position Accuracy & $10 \%$ \\
\hline 4 & Visual Representation Accuracy & $15 \%$ \\
\hline 5 & On-Route Guidance & $0 \%$ \\
\hline 6 & Purchase Order/Payment Accuracy & $15 \%$ \\
\hline 7 & Product Movement Activeness & $0 \%$ \\
\hline
\end{tabular}

Table 1: Performance evaluation criteria for sales representatives of Beverage company Sales department

Further, the organization was asked to provide an integer from 60 to 100 when creating an overall performance rating for each employee. This was to bring about uniformity in rating and for psychological analysis. Instructions with regard to possible bias against individuals who had been in the job for a shorter or longer period of time was made clear to the performance rater. The final ratings were then determined and mutually agreed upon by the organization and Harrison Assessments to be used for analysis.

Performance scoring format is given to table 2 for the sake of clarity.

\begin{tabular}{|l|l|}
\hline Score & Category \\
\hline 100 & Ideal \\
\hline 90 & Excellent \\
\hline 80 & Good \\
\hline 75 & Average \\
\hline 70 & Below Average \\
\hline 60 & Poor \\
\hline
\end{tabular}

Table 2: Performance evaluation scale of sales depart of the Beverage Company

\section{Procedure}

The performance benchmarking is conducted by Harrison Assessments in different phases as given in figure2. Each individual went through this process which comprised of a pre-assessment phase and three benchmarking phases. These phases are described below and the entire process was done in a span of 4 months.

- Pre-assessment Phase was to identify the scope, viability and adequacy of data \& information to be collected. To identify possible controlled variables and identify variables beyond control

- Data Collection \& Review

o Collection of documents on job descriptions and Key Performance Indicators

o Collection of information on job performance from the supervisor of the employee (sales)

o Collection of details of each member of the sample

o Data Cleansing 
- Harrison Assessments Profiling and Data Input

o Communication to each member of the sample to respond to the online questionnaire

o Employees who completed the questionnaires are accepted to the analysis of the study (this is determined by the consistency score secured by each individual).

o Categorization of the data as per Harrison Assessments benchmarking methodology

o Key-in Performance ratings for each member of the sample in the Harrison Assessments system

- Analysis and Presentation

o The data was loaded into Harrison Assessments International's analysis software for further analysis using Automatic Benchmarking technology

o Identify traits that were likely to be associated with Job Performance

o Preparation and presentation of each individual's and group profile.

\section{RESULTS AND DISCUSSION}

The data collected was analysed qualitatively and quantitatively. Both descriptive and inferential statistics were used for analysis. The entire sample was assessed through Harrison Assessments Questionnaire and each member's job performance data was ascertained.

The employees in the sample group were run against the resulting Job Success Formula template. The mean suitability score was 82.23 with a standard deviation of 9.77.

Out of the 175 Harrison Assessments' traits, 39 traits showed a relationship with job performance. Ten of the 39 traits showed a significant linear correlation with performance and thus were included as essential traits. The correlations of the essential traits ranged between 0.289 and 0.100 . (for the sake of clarity, Operational definition of these 10 traits is explained in Appendix A.) The correlations of the essential traits are shown below:

As part of analysis essential traits for the sales representative position are drawn are presented below:

\begin{tabular}{|l|l|l|l|l|}
\hline Essential Trait & Mean & SD & Coefficient & $\begin{array}{l}\text { Significance } \\
\text { level }\end{array}$ \\
\hline Ambitious Benevolence & 7.97 & 1.27 & 0.276 & 0.0181 \\
\hline Wants High Pay & 7.79 & 2.30 & 0.289 & 0.0131 \\
\hline Wants Frankness & 6.71 & 2.06 & 0.210 & 0.0738 \\
\hline Compassionate Enforcing & 6.82 & 1.24 & 0.167 & 0.1575 \\
\hline Systematic & 6.59 & 1.50 & 0.187 & 0.1125 \\
\hline Warmth / empathy & 6.80 & 1.99 & 0.157 & 0.1849 \\
\hline Planning & 7.32 & 1.65 & 0.113 & 0.3397 \\
\hline Frank & 7.87 & 1.71 & 0.161 & 0.1743 \\
\hline Helpful & 7.83 & 1.29 & 0.118 & 0.3203 \\
\hline Tempo & 5.66 & 1.67 & 0.100 & 0.3979 \\
\hline
\end{tabular}

Table3: Trait list with Correlation Coefficient 
Further analysis was conducted to arrive at a score with a combination of 10 essential traits (mentioned above) along with other desirable traits. As a consequence, for the present study 39 traits were finally grouped and analysed and the benchmarked template was framed. This template has helped in mapping suitability score for each individual. Average score was 82.23. The overall suitability score automatically generated from the job success formula template on suitability and the correlation coefficient with performance was 0.633 . The overall Suitability Score generated for Sales Representatives had a 0.633 correlation with actual performance.

\begin{tabular}{|l|l|l|l|l|l|}
\hline & $\mathbf{N}$ & Mean & SD & Coefficient & $\begin{array}{l}\text { Significance } \\
\text { Level }\end{array}$ \\
\cline { 2 - 6 } Suitability Score & 73 & 82.23 & 9.77 & 0.633 & 0.0000 \\
\hline
\end{tabular}

Table 4: Correlation Coefficient Suitability Score and Performance Score

The group of traits that are drawn as essential traits are the ones that appeared to be influencing the high job performance. The author would like to state certain details regarding these traits keeping in view the beverage company (leading soft drink manufacturer) dealing with a product that is related to food industry, is a perishable product and has a direct impact on consumers health and pleasure.

Sales job has ambition inherently built into it and in this case there are set targets and also has a bearing on consumers health. They have to keep in mind targeted selling i.e. before the expiry date so that it will not harm the consumer. This justifies the presences of trait Ambitious benevolence (refer to table 3)

It may be noted that the trait Wants High Pay and Tempo goes together for a sales position. As often we see that a person who aspire for High pay takes up sales position even though there is a certain amount of risk interms of travel, being away from family, untimely meals, sleep deprivation etc. The salary may be fixed but often incentives are high and incentives depend on their ability to close the deal at a faster pace which reflect Tempo (refer to table 3).

Sales job often requires a quick understanding on the spot from a customer's need inorder to start or close the deal. Expression of frankness from a customer is often appreciated because that helps in their purchasing decisions regarding the nature of the product which they are selling and also the competitors in the market. Sales person's ability to be frank without being harsh and rude in the process of selling will go a long way in customer satisfaction and customer loyalty to the product. Hence Frankness is recommended (refer to table 3).

Being Systematic and Planning well is needed to conduct the business in a very professional manner. This could help in keeping up the image of the company and contributes to the timely revenue (refer to table 3). 
The traits 'Compassionate enforcing', 'Warmth/ Empathy', 'Helpful' go together in the field of selling as they need to understand and accept individual differences, different levels of abilities, different backgrounds, differences in knowledge levels of product of various clients and the team members. Unless and until they help them to understand and be empathetic he/she will not be a successful sales person. This is important in this context as a food product and soft drinks are facing stiff competition in the market and sensitive to customer's demands, expectations and their food preferences (refer to table 3).

\section{CONCLUSION}

- The results of this study provided insight into the combination of traits that relate to performance within the sales representative position for the company.

- The benchmarking technology produced a job success formula template with an average suitability score of 82.23 and correlation of 0.633

- The analysis resulted in identifying 39 traits related to job success including the traits that hinder performance.

- A total of 10 essential traits that would impact performance along with correlations were identified

- The results of this validation study demonstrated a statistically significant relationship between the overall suitability scores produced by the job success formula template and the performance scores.

- Factors such as eligibility; Education and skills were not taken into consideration inorder to make sure that suitability scores were not biased

\section{OUTCOME}

- The results were shared with the company's sales executive team. The template was accepted and decision to use it for forthcoming recruitment process for screening candidates was made a policy

- $\quad$ The template that emerged from the current study can be used for other sales position in the field of food and beverage manufacturing companies if they desire.

\section{REFERENCES:}

Ajelabi I \& Tang Y (2010), “The Adoption of Benchmarking Principles for Project Management Performance Improvement,”, International Journal of Managing Public Sector Information and Communication Technologies (IJMPICT) Vol. 1, No. 2, December 2010.

APQC (1993). Basics of Benchmarking, (Houston, Texas: APQC,). Kazmi A (2007). "Business Policy and Strategic Management”, Tata McGraw-Hill, New Delhi, 2e, pp. 154; 408; 438.

Bhutta K.S Huq F. (1999) Benchmarking best practices: an integrated approach. Benchmarking: An international Journal, 6 (3), 254-268. 
Camp R (1989). The search for industry best practices that lead to superior performance, Productivity Press.

Chadha P (2007). "Performance Management”, MACMILLAN, New Delhi pp. 76-77.

Churchill, G. A, Ford, N. M, Hartley, S.W, Walker, O.C (1985) The determinants of salesperson Performance: A Meta Analysis. Journal of Marketing Research, 22, 103-118.

Kyro P.(2003) Revising the concepts and forms of benchmarking. Benchmarking: An international Journal , 10(3), 210-225.

Savanam C (2010). "Benchmarking”, African Journal of Business Management Vol. 4(6), pp. 882-885, June 2010.

\section{APPENDIX A}

ESSENTIAL TRAITS - These are traits in which higher scores generally relate better performance.

Ambitious Benevolence - The desire to help society through developing and using one's own financial strength

Wants High Pay - The desire to earn greater remuneration

Wants Frankness - The desire for others to be direct, straightforward, and to the point

Compassionate Enforcing - The tendency to enforce necessary rules with compassion

Systematic - The enjoyment of tasks that require carefully or methodically thinking through steps.

Warmth / empathy - The tendency to express positive feelings and affinity toward others

Planning - The tendency to formulate ideas related to the steps and process of accomplishing an objective

Frank - The tendency to be straightforward, direct, to the point, and forthright

Helpful - The tendency to respond to others' needs and assist or support others to achieve their goals

Tempo - The enjoyment of work that needs to be done quickly

DESIRABLE TRAITS - These are traits in which low scores hinder success but there is not necessarily a significant overall correlation with performance. In other words, poor performers have a higher incidence of low scores on this type of trait.

Repetition - The tolerance of monotonous work: a single activity repeated over and over (e.g. assembly line)

Team - The enjoyment of working closely in a co-operative team effort (not necessarily the ability to do so)

Wants Diplomacy - The desire for others to be tactful

Pressure Tolerance - The level of comfort related to working under deadlines and busy schedules Wants Autonomy - The desire to have freedom or independence from authority

Wants To Lead - The desire to be in a position to direct or guide others

Comfort With Conflict - The tendency to be comfortable with confrontation or strife 
Enthusiastic - The tendency to be eager and excited toward one's own goals

Self-acceptance - The tendency to like oneself ("I'm O.K. the way I am")

Influencing - The tendency to try to persuade others

Tolerance Of Bluntness - The level of comfort related to receiving abrupt or frank communications from others

Experimenting - The tendency to try new things and new ways of doing things

Numerical - The enjoyment of counting, calculating, or analyzing quantities using mathematics

Enforcing - The tendency to insist upon necessary rules being followed

Public Speaking - The enjoyment of presenting or articulating information to groups of people

TRAITS TO AVOID - These are traits that hinder success but don't necessarily have a negative correlation with success.

Narrowly Unambitious - The tendency to lack of motivation to help society while at the same time lacking motivation for financial gain

Stubborn Persistence - The tendency to tenaciously pursue the same course of action without experimenting with different ways of accomplishing the objective

Blindly Optimistic - The tendency to focus on the possible benefits of a plan or strategy, while failing to adequately see the potential difficulties

Impulsive - The tendency to take risks without sufficient analysis of the potential difficulties

Precise But Slow - The tendency to be exact but not paying sufficient attention to productivity

Skeptical - The tendency to overly emphasize the potential difficulties of a plan or strategy without giving sufficient emphasis to the potential benefits

Cool Permissiveness - The tendency to lack warmth while at the same time avoiding enforcing necessary rules

Self-critical - Disliking oneself in the context of self-improvement

Insensitive - The tendency to be assertive with one's own needs while lacking sufficient warmth for others

Inconclusive - The tendency to lack certainty in ones opinions while at the same time being very open to the ideas of others

Cautious Inattention - The tendency to be cautious about risks while at the same time paying little attention to the potential pitfalls of a plan or strategy

Dominating - The tendency to be assertive with one's own needs while failing to respond to other people's needs

Self-sacrificing - The tendency to respond to others' needs at the expense of one's own needs Defers Decisions - The tendency to avoid making decisions by referring them to others

\footnotetext{
How to cite this article: S Gajula, B Chintalapuri (2016), To Arrive at Benchmarking for through Evaluation of Job Performance and Behavioural Assessment, International Journal of Indian Psychology, Volume 3, Issue 3, No. 6, DIP: 18.01.114/20160303
} 